\title{
AUSLANDER-REGULAR ALGEBRAS AND MAXIMAL ORDERS
}

\author{
J. T. STAFFORD
}

\begin{abstract}
Let $R$ be an Auslander-regular, Cohen-Macaulay, Noetherian ring that is stably free. Then, we prove that $R$ is a domain and a maximal order in its division ring of fractions. In particular, this applies to the Sklyanin algebra $S$ and shows that, when $S$ satisfies a polynomial identity, it is actually a finite module over its centre.
\end{abstract}

\section{Introduction}

Fix once and for all a field $k$. Throughout this paper, all rings will be $k$-algebras. Following, for example, [13], a ring $R$ is called Auslander-regular provided that $R$ has finite global homological dimension and satisfies the Gorenstein condition: if $p<q$ are non-negative integers and $M$ is a finitely generated $R$-module, then $\operatorname{Ext}_{R}^{p}(N, R)=0$ for every submodule $N$ of $\operatorname{Ext}_{R}^{q}(M, R)$. Set

$$
j(M)=\min \left\{r: \operatorname{Ext}^{r}(M, R) \neq 0\right\}
$$

and write GK-dim $(M)$ for the Gelfand-Kirillov dimension of $M$. Then, an Auslander-regular ring $R$ is called Cohen-Macaulay (which will be abbreviated to $\mathrm{CM}$ ), provided that GK-dim $(R)<\infty$ and $j(M)+\mathrm{GK}-\operatorname{dim}(M)=\mathrm{GK}-\operatorname{dim}(R)$ holds for every finitely generated $R$-module $M$. A ring $A$ is called stably free if, for every finitely generated projective $A$-module $P$, there exist integers $n$ and $m$ such that $P \oplus A^{(n)} \cong A^{(m)}$. The aim of this paper is to prove the following.

THEOREM (see Theorem 2.10). Let $R$ be a Noetherian $k$-algebra that is Auslanderregular and CM. Assume that $R$ is also stably free. Then, $R$ is a domain and a maximal order in its quotient division ring $Q(R)$; that is, if $R \subseteq S$ for some ring $S$ with the property that $a S b \subseteq R$, for some $a, b \in R \backslash\{0\}$, then $S=R$.

The proof that $R$ is a domain is very similar to the proof given in [13] for graded rings, and so the main point of the paper is to prove that $R$ is a maximal order. This is proved in Section 2, while various homological results needed for the proof are given in Section 1. The hypothesis that $R$ be stably free can be considerably weakened; indeed, for a prime ring $R$ one can replace the assumption that $R$ is stably free by the hypothesis that the state space of $R$ is trivial (the state space is defined in Section 2, after Lemma 2.8).

One of the author's main motivations in proving the Theorem is for applications to the regular rings defined in [2]. These are defined as follows. For the purposes of

Received 15 February 1992.

1991 Mathematics Subject Classification 16A18.

This research was supported in part by an NSF grant. 
this paper, a graded algebra $A$ is defined to be a (connected) $\mathbb{N}$-graded algebra, generated in degree one; thus $A=\bigoplus_{i \geqslant 0} A_{i}$, where $A_{0}=k$ is central, $\operatorname{dim}_{k} A_{i}^{i}<\infty$ for all $i$, and $A$ is generated as an algebra by $A_{1}$. Let $A^{+}=\bigoplus_{i \geqslant 1} A_{i}$ denote the graded radical of a graded ring $A$. Then an Artin-Schelter regular ring of dimension $d$ is defined to be a graded ring $R$ of global dimension $d$, written $\operatorname{gldim}(R)=d$, such that $R$ has finite Gelfand-Kirillov dimension and satisfies a weak form of the Gorenstein property: if $k=R / R^{+}$, then

$$
\operatorname{Ext}_{R}^{n}\left(k_{R}, R\right)=\delta_{n d} \cdot\left({ }_{R} k\right)
$$

In dimensions $d=3$ and $d=4$, there exist nontrivial examples of Noetherian, Artin-Schelter regular rings, $S=S(E, \tau)$, arising from an elliptic curve $E \subset \mathbb{P}^{d-1}$ and a point $\tau$ on $E$; see $[3,4,17,18]$. (The reader should be warned that the notation $S(E, \tau)$ is only being used for the purposes of this Introduction, since [18] shows that there can exist more than one algebra $S$ associated to a given pair $\{E, \tau\}$.) For each of these algebras, it follows from [4] and [13] that the ring is Auslander-regular and CM, while any graded ring is clearly stably free. Thus, the Theorem implies that all these examples of Artin-Schelter regular rings are maximal orders.

This has other consequences for the structure of the ring. In [4], the authors proved that, for the Artin-Schelter regular rings of dimension three:

(i) $S=S(E, \tau)$ satisfies a polynomial identity (PI) if and only if $\tau$ is a point of finite order,

(ii) if $S$ is PI then it is actually a finite module over its centre.

In [16] Smith studied the representation theory of the Sklyanin algebras $S\left(\alpha_{1}, \alpha_{2}, \alpha_{3}\right)$; that is, the algebras proved to be Artin-Schelter regular in [17]. Using methods similar to those in [4], he was able to prove that (i) holds also for the Sklyanin algebras, but was unable to prove (ii). However, this follows trivially from the Theorem.

COROLlaRY. Let $R$ be as in the Theorem. If $R$ is PI and finitely generated as a $k$-algebra, then $R$ equals its trace ring, in the sense of [14, Section 13.9]. Moreover, $R$ is a finite module over its centre $Z(R)$, and $Z(R)$ is an integrally closed, Noetherian domain.

In particular, a Sklyanin algebra $S\left(\alpha_{1}, \alpha_{2}, \alpha_{3}\right)$ is $\mathrm{PI}$ if and only if it is a finite module over its centre.

As remarked in [1], the methods of $[3,4]$ can also be used to prove that the Artin-Schelter regular, PI rings of dimension three are maximal orders and this has applications to the study of Brauer groups. However, Artin's proof is rather indirect and specific to these rings. This led the author to prove the present Theorem, and hopefully it will also have applications to the theory of Brauer groups, along the lines of [1].

The Theorem has an unfortunately large number of hypotheses. However, for a Noetherian domain $R$ of finite global dimension, it is easy to give examples to show that the Theorem will fail if either $R$ is not Auslander-regular or if $R$ does not have a trivial state space (see Section 3 ). Thus, the only obvious improvement that could be made in the Theorem is to remove the CM hypothesis. For a graded Noetherian ring $R$, however, it is an open question whether the possession of finite global dimension is sufficient to force $R$ to be a domain and a maximal order. $\dagger$ As slight

$\dagger$ This question has now been answered for PI rings (see note at end of paper). 
evidence for this conjecture, we show that it is true when $R$ is integral over its centre. This is proved in Proposition 2.12 and follows easily from the corresponding results for local rings proved in $[8,10]$.

\section{Homological algebra}

In this short section we prove several results on the Gelfand-Kirillov dimension of homology groups that will be required in proving the main theorem. Gelfand-Kirillov dimension is called exact for $R$ if, for any $R$-modules $M^{\prime} \subseteq M$ one has

$$
\mathrm{GK}-\operatorname{dim}(M)=\max \left\{\mathrm{GK}-\operatorname{dim}\left(M^{\prime}\right), \mathrm{GK}-\operatorname{dim}\left(M / M^{\prime}\right)\right\} .
$$

Given an $R$-T-bimodule $M$, for rings $R$ and $T$, then $M$ is called a finitely generated $R$-T-bimodule provided that $M$ is finitely generated as both a left $R$-module and a right $T$-module. Note that, in this situation, the Gelfand-Kirillov dimensions of $M$ as a module over $R$ and $T$ are equal (see [11, Corollary 5.4]), and so we may denote it by $\mathrm{GK}(M)$ without ambiguity. However, this equality will in general fail if $M$ is not finitely generated on both sides, in which case we shall write $\ell$-GK-dim $(M)$ and $r$-GK-dim $(M)$ for the Gelfand-Kirillov dimensions of ${ }_{R} M$, respectively, $M_{T}$.

In this section we shall make frequent use of the following spectral sequence. Let $R, U$ and $T$ be $k$-algebras such that $R$ is a Noetherian ring of finite global dimension. Suppose that $M$ is an $R$ - $U$-bimodule, while $N$ is an $R-T$-bimodule, such that $N$ is a finitely generated $R$-module. Then there is a spectral sequence

$$
\operatorname{Tor}_{p}^{R}\left(\operatorname{Ext}_{R}^{q}(M, R), N\right) \Rightarrow \operatorname{Ext}_{R}^{q-p}(M, N) .
$$

Observe that $\operatorname{Ext}_{R}^{q}(M, R)$ carries a natural $U$-R-bimodule structure and so each term on the left or right of (1.1) is therefore a $U$-T-bimodule. Moreover, all of the morphisms that are constructed between the terms of the spectral sequence are $U$-T-bimodule homomorphisms. In particular, $\operatorname{Ext}_{R}^{q-p}(M, N)$ is a subfactor of

$$
\bigoplus \operatorname{Tor}_{p+j}^{R}\left(\operatorname{Ext}_{R}^{q+j}(M, R), N\right)
$$

as $U$-T-bimodules. While the spectral sequence (1.1) is well-known as a spectral sequence for finitely generated $R$-modules, the author can find no reference to substantiate the claim made above that it works equally well for bimodules, so we shall indicate why this holds. The proof that (1.1) is a spectral sequence (for finitely generated $R$-modules) is given in some detail in [5, pp. 56-58], so we shall simply indicate the modifications that need to be made to Bjork's proof. To begin with, note that $R$ - $U$-bimodules may be identified with right $U \otimes_{k} R^{\text {op }}$-modules. Thus, by [9, Corollary 9.2.4], there exists a finite projective resolution, say $P$, of ${ }_{R} M$ that is a complex of $R$ - $U$-bimodules; that is, each object $P^{j}$ is an $R$ - $U$-bimodule and the maps $P^{j} \rightarrow P^{j-1}$ are $R$ - $U$-bimodule homomorphisms. Similarly, let $Q^{\cdot}$ be a complex of $R$ - $T$-bimodules that is a projective resolution of ${ }_{R} N$. Of course, the $Q^{i}$ and $P^{j}$ need not now be finitely generated $R$-modules but by adding an appropriate free module to each term, we may assume that each $P^{j}$ is a free $R$-module, say $P^{j} \cong \oplus R_{i}$, where each $R_{i} \cong R$. Next, there exists a natural homomorphism of $U$-T-bimodules

$$
\phi: \operatorname{Hom}_{R}\left(P^{j}, R\right) \otimes_{R} N \longrightarrow \operatorname{Hom}_{R}\left(P^{j}, N\right),
$$

given by $\theta \otimes n \rightarrow\{p \rightarrow \theta(p) n\}$. We claim that this is an isomorphism, for which it suffices to prove that it is an isomorphism of abelian groups. But, as such, $\phi$ is just 
the composition of the following maps (recall that $R$ is Noetherian and ${ }_{R} N$ is finitely presented):

$$
\begin{aligned}
\operatorname{Hom}_{R}\left(P^{j}, R\right) \otimes N & \cong\left\{\left(\operatorname{Hom}\left(R_{i}, R\right)\right)\right\} \otimes N & & \text { (using [15, Theor } \\
& \cong \prod\left\{\operatorname{Hom}\left(R_{i}, R\right) \otimes N\right\} & & \text { (by [9, Exercise } \\
& \cong \prod\left(\operatorname{Hom}\left(R_{i}, N\right)\right) \cong \operatorname{Hom}\left(P^{j}, N\right) & & \text { (by [15], again). }
\end{aligned}
$$

Thus, $\phi$ is indeed an isomorphism. Since $R$ is coherent,

$$
\operatorname{Hom}\left(P^{j}, R\right) \cong \prod\left(\operatorname{Hom}\left(R_{i}, R\right)\right)
$$

is a direct product of flat modules and therefore is itself flat [15, Theorem A, p. 113]. Hence the double complex at the top of [5, p. 57] does still have exact columns. The proof given in [5] now goes through without change, except that now all morphisms are $U$-T-bimodule homomorphisms. Thus (1.1) is, indeed, a spectral sequence of $U$ - $T$-bimodules.

LEMMA 1.2. Let $R, U$ and $T$ be Noetherian rings and suppose that $M$ is a finitely generated $U$-R-bimodule, while $N$ is a finitely generated $R$-T-bimodule. Then, for all $j \geqslant 1, \mathrm{GK}-\operatorname{dim}\left(\operatorname{Tor}_{j}^{R}(M, N)\right) \leqslant \min \{\mathrm{GK}-\operatorname{dim}(M), \mathrm{GK}-\operatorname{dim}(N)\}$.

REMARK. The proof of the lemma also shows the well-known fact that $\operatorname{Tor}_{j}^{R}(M, N)$ is a finitely generated $U$-T-bimodule, and so the statement of the lemma does make sense.

Proof. Pick a finitely generated, projective resolution, say $F^{*}$, of $N$ as a left $R$-module. Then $M \otimes_{R} F^{\cdot}$ is a complex of left $U$-modules, and $\operatorname{Tor}_{j}^{R}(M, N)$ is a subfactor of $M \otimes F^{j}$, as left $U$-modules. In particular, this implies that $\operatorname{Tor}_{j}^{R}(M, N)$ is a finitely generated $U$-module and, dually, $\operatorname{Tor}_{j}^{R}(M, N)$ is a finitely generated right $T$-module. But, again as left $U$-modules, $M \otimes F^{j} \cong M^{(n)}$, for some $n$. Thus $\ell-\mathrm{GK}-\operatorname{dim}\left(\operatorname{Tor}_{j}^{R}(M, N)\right) \leqslant \mathrm{GK}-\operatorname{dim}(M)$. The same argument works on the other side.

Proposition 1.3. Let $R, U$ and $T$ be k-algebras, such that $R$ is a Noetherian ring of finite global dimension. Suppose that $M$ is an $R$-U-bimodule and $N$ is an $R$-T-bimodule such that both are finitely generated as $R$-modules. Fix non-negative integers $n$ and $\alpha$. Assume that:

(i) Gelfand-Kirillov dimension is exact for $U$-modules;

(ii) $r-\mathrm{GK}-\operatorname{dim}_{R}\left(\operatorname{Ext}_{R}^{q}(M, R)\right) \leqslant \alpha$ for all $q \neq n$. Then:

(A) $\ell-G K-\operatorname{dim}_{U}\left(\operatorname{Tor}_{p}^{R}\left(\operatorname{Ext}_{R}^{n}(M, R), N\right)\right) \leqslant \alpha$ for all $p>n$;

(B) suppose that $\ell-\mathrm{GK}-\operatorname{dim}_{U}\left(\operatorname{Ext}_{R}^{n-m}(M, N)\right)=\beta>\alpha$, for some $\beta$ and $m \in \mathbb{Z}$, then $\ell-\mathrm{GK}-\operatorname{dim}_{U}\left(\operatorname{Tor}_{m}^{R}\left(\operatorname{Ext}_{R}^{n}(M, R), N\right)\right) \geqslant \beta$;

(C) suppose that $\operatorname{Ext}_{R}^{n}(M, R)$ is a finitely generated $U$-R-bimodule and that $N$ is a finitely generated $R-T$-bimodule, then parts $(\mathrm{A})$ and $(\mathrm{B})$ hold with $\ell-\mathrm{GK}$ - $\operatorname{dim}_{U}$ replaced by $r$-GK-dim ${ }_{T}$.

REMARK. We shall use this result only in the situation that $R=U=T$, but distinguishing the three rings makes the various module actions somewhat clearer. In fact, we shall apply this when $R$ is an Auslander-regular, CM domain. In these circumstances, (i) will always hold and (ii) holds if, for example, $M$ is torsion-free $R$-bimodule, $n=0$ and $\alpha=\mathrm{GK}$ - $\operatorname{dim}(R)-2$ (see Lemmas 2.1 and 2.3 , respectively). 
Proof. Set $E^{q}=\operatorname{Ext}_{R}^{q}(M, R)$ and $T^{p q}=\operatorname{Tor}_{p}^{R}\left(\operatorname{Ext}_{R}^{q}(M, R), N\right)$. As $M$ is a finitely generated left $R$-module, each $E^{q}$ is a finitely generated right $R$-module and $r$-GK-dim $\left(E^{q}\right) \leqslant \alpha$ for each $q \neq n$. Unfortunately, the $E^{q}$ need not be finitely generated as left $U$-modules. Nonetheless, $\ell$-GK- $\operatorname{dim}_{v}\left(E^{q}\right) \leqslant r-\mathrm{GK}-\operatorname{dim}_{R}\left(E^{q}\right) \leqslant \alpha$ does hold for all $q \neq n$ (see [11, Lemma 5.3]). Now, consider $T^{p q}$, for some $q \neq n$. As in the last lemma, pick a finitely generated, free resolution, say $Q^{\circ}$ of ${ }_{R} N$ and suppose that $Q^{p}$ has rank $t$. Then, as left $U$-modules, $T^{p q}$ is a subfactor of $E^{q} \otimes Q^{p} \cong\left(E^{q}\right)^{(t)}$. Thus, $\ell$-GK-dim $\left(T^{p q}\right) \leqslant \ell$-GK-dim $\left(E^{q}\right) \leqslant \alpha$ for all $q \neq n$.

(A) Now apply the spectral sequence (1.1), considering each term as a left $U$-module. Then, modulo modules of left Gelfand-Kirillov dimension at most $\alpha$, the spectral sequence collapses. In other words, in the ' $E_{p q}^{2}$ table (as, for example, written out in [5, p. 57]), only the terms in the $n$th column, the $T^{p n}$, can possibly have left Gelfand-Kirillov dimension greater than $\alpha$. Fix $p>n$. In passing to the limit of the spectral sequence, one obtains bisubmodules $X \subseteq Y \subseteq T^{p n}$ such that $Y / X \hookrightarrow \operatorname{Ext}_{R}^{n-p}(M, N)$. Here, $X$ has a chain of bisubmodules

$$
X=X_{s} \supseteq X_{s-1} \supseteq \ldots \supseteq X_{0}=0,
$$

where each factor $X_{u} / X_{u-1}$ is a subfactor of $T^{t q}$, for some $t \geqslant 0$ and $q>n$. By the last paragraph, these subfactors satisfy $\ell$-GK-dim $\left(X_{u} / X_{u-1}\right) \leqslant \alpha$. Thus, exactness implies that $\ell$-GK-dim $(X) \leqslant \alpha$. Similarly,

$$
\bar{Y}=T^{p n} / Y=Y_{v} \supseteq Y_{v-1} \supseteq \ldots \supseteq Y_{0}=0
$$

for some bisubmodules $Y_{u}$ with the property that each $Y_{u} / Y_{u-1}$ is a subfactor of $T^{t q}$, for some $t \geqslant 0$ and $q<n$. Thus, once again, $\ell$-GK-dim $(\bar{Y}) \leqslant \alpha$. But, in the limit, $Y / X \hookrightarrow \operatorname{Ext}_{R}^{n-p}(M, N)=0$. Thus, $X=Y$ and exactness implies that

$$
\ell-\mathrm{GK}-\operatorname{dim}\left(T^{p n}\right) \leqslant \max \{\ell-\mathrm{GK}-\operatorname{dim}(\bar{Y}), \ell-\mathrm{GK}-\operatorname{dim}(X)\} \leqslant \alpha,
$$

as required.

(B) In this case, the spectral sequence shows that (as $U$-T-bimodules) $\operatorname{Ext}_{R}^{n-m}(M, N)$ is a subfactor of $\bigoplus_{i \in \mathbb{Z}} T^{i, n-m+i}$. But, by hypothesis, $\ell$-GK$\operatorname{dim}_{U}\left(\operatorname{Ext}_{R}^{n-m}(M, N)\right)=\beta>\alpha$. Therefore, by the comments of the first paragraph of the proof, $\ell$-GK- $\operatorname{dim}_{v}\left(T^{m n}\right) \geqslant \beta$, as required.

(C) Finally, consider the case when $E^{n}$ and $N$ are finitely generated bimodules. Then each $T^{p n}=\operatorname{Tor}_{p}^{R}\left(\operatorname{Ext}_{R}^{n}(M, R), N\right)$ is also a finitely generated $U$-T-bimodule. Thus, [11, Corollary 5.4] implies that $r-\mathrm{GK}-\operatorname{dim}\left(T^{p n}\right)=\ell-\mathrm{GK}-\operatorname{dim}\left(T^{p n}\right)$ for each $p$.

\section{Maximal orders}

In this section, we prove the Theorem and Corollary from the Introduction. Thus, throughout the section we assume that $R$ is a Noetherian prime ring, finitely generated as a $k$-algebra and such that $R$ is Auslander-regular and Cohen-Macaulay. Set GK-dim $(R)=\mu$.

Let $M$ be a finitely generated (right) $R$-module. Then $M$ is called $p$-pure if $j(N)=p$ for all non-zero submodules $N$ of $M$. Since $R$ is CM, $M$ is $p$-pure if and only if $M$ is $(\mu-p)$ homogeneous in the sense that $\mathrm{GK}-\operatorname{dim}(N)=\mu-p$ for all non-zero submodules $N$ of $M$. For convenience, we shall write

$$
\begin{gathered}
E^{p}\left(M_{R}\right)=\operatorname{Ext}^{p}(M, R), \\
E^{p q}\left(M_{R}\right)=\operatorname{Ext}^{p}\left(\operatorname{Ext}^{q}(M, R), R\right)=E^{p}\left({ }_{R} E^{q}(M)\right) .
\end{gathered}
$$


As usual, the subscripts will be dropped whenever there is no possible confusion. When $p=q=0$, we shall denote $E^{0}\left(M_{R}\right)$ and $E^{00}\left(M_{R}\right)$ by $M_{R}^{*}$ and $M_{R}^{* *}$, respectively. The module $M$ is called (right) reflexive if the natural map $M \rightarrow M^{* *}$ is an isomorphism. Equivalently, $M$ is reflexive if and only if $M={ }_{R} Y^{*}$, for some finitely generated, left $R$-module $Y$. Write $Q(R)$ for the simple artinian quotient ring of $R$. If $M$ is an essential right $R$-submodule of $Q(R)$, then we shall always identify $M_{R}^{*}$ with $\{q \in Q(R): q M \subseteq R\}$. Similarly, $\operatorname{End}_{R}(M)$ will be identified with

$$
\mathcal{O}_{\ell}(M)=\{q \in Q(R): q M \subseteq M\} .
$$

Similar definitions will be used for left $R$-modules.

We begin with three results from the literature that will be used frequently.

\section{LEMMA 2.1. Gelfand-Kirillov dimension is exact for $R$-modules.}

Proof. Levasseur shows in [13, Proposition 5.9] that Gelfand-Kirillov dimension is exact for finitely generated $R$-modules, but this is sufficient to prove it for all modules. To see this, let $M^{\prime} \subseteq M$ be right $R$-modules, and let $U$ be a finitely generated submodule of $M$. Then, by exactness for finitely generated modules,

$$
\begin{aligned}
\mathrm{GK}-\operatorname{dim}(U) & =\max \left\{\mathrm{GK}-\operatorname{dim}\left(\left(U+M^{\prime}\right) / M^{\prime}\right), \mathrm{GK}-\operatorname{dim}\left(U \cap M^{\prime}\right)\right\} \\
& \leqslant \max \left\{\mathrm{GK}-\operatorname{dim}\left(M / M^{\prime}\right), \mathrm{GK}-\operatorname{dim}\left(M^{\prime}\right)\right\} .
\end{aligned}
$$

Hence, GK-dim $(M) \leqslant \max \left\{\mathrm{GK}-\operatorname{dim}\left(M / M^{\prime}\right), \mathrm{GK}-\operatorname{dim}\left(M^{\prime}\right)\right\}$, as required.

THEOREM 2.2 (Gabber's Theorem). If $M$ is a finitely generated, p-pure right $R$-module, then $M \subseteq E^{p p}(M)$, as right $R$-modules, and $E^{p p}(M)$ is the unique, largest essential extension $M^{\prime}$ of $M$ such that $j\left(M^{\prime} / M\right) \geqslant p+2$. Moreover, $E^{p p}(M)$ is a finitely generated right $R$-module.

Thus, if $M$ is a finitely generated right $R$-submodule of $Q(R)$, then $M_{R}^{* *}=E^{00}(M)$ can (and will) be identified with the unique, largest, $R$-submodule $M^{\prime}$ of $Q(R)$ such that $M \subseteq M^{\prime}$ and $\mathrm{GK}-\operatorname{dim}\left(M^{\prime} / M\right) \leqslant \mu-2$.

Proof. See, for example, [6, Theorem 3.6 and Example 3.2].

LEMMA 2.3. Let $M$ be a finitely generated, p-pure right $R$-module. Then $E^{p}(M)$ and $E^{p p}(M)$ are also p-pure. Moreover, GK-dim $\left(E^{q}(M)\right) \leqslant \mu-q-1$ for all $q>p$. If $M=E^{p p}(M)$, then GK-dim $\left(E^{q}(M)\right) \leqslant \mu-q-2$ for all $q>p$. 3.5].

Proof. See [6, Proposition 2.11, Theorem 2.12, Definition 3.3 and Proposition

One of the main reasons for needing to assume that $R$ is CM as well as Auslanderregular, is to provide symmetry; if $M$ is a finitely generated $R$-bimodule, then $j\left({ }_{R} M\right)=\mu-\mathrm{GK}-\operatorname{dim}(M)=j\left(M_{R}\right)$. If $R$ were not CM, then there would be no reason for this to hold. As is shown in the next few corollaries, this symmetry gives one a strong control on the reflexive $R$-modules.

COROllary 2.4. Suppose that $X$ is a right $R$-submodule of $Q(R)$ such that $R \subset X$ (throughout $\subset$ is used in its strict sense). Then $\mathrm{GK}-\operatorname{dim}(X / R)=\mu-1$. 
Proof. It suffices to prove the result when $X_{R}$ is finitely generated. If GK-dim $(X / R) \leqslant \mu-2$, then, by Gabber's Theorem, $X \subseteq R_{R}^{* *}=R$, a contradiction.

COROLlARY 2.5. Suppose that $M$ is a non-zero, finitely generated R-bisubmodule of $Q(R)$ that is essential as a right submodule. Then:

(i) if $X$ is a finitely generated, right $R$-submodule of $M_{R}^{*}$, then $X_{R}^{* *} \subseteq M_{R}^{*}$;

(ii) $M_{R}^{* *}={ }_{R} M^{* *}$; in particular, $M_{R}$ is reflexive if and only if ${ }_{R} M$ is reflexive.

REMARK. Part (ii) of Corollary 2.5 proves, in particular, that $M_{R}^{* *}$ is a finitely generated $R$-bimodule. We leave as an exercise the following generalization. If $M$ is a finitely generated $R$-bimodule that is right $p$-pure for some integer $p$, then $M$ is left $p$-pure and $E^{p p}\left(M_{R}\right)=E^{p p}\left({ }_{R} M\right)$ is a finitely generated $R$-bimodule.

Proof. (i) Write $M^{*}=M_{R}^{*}$ and $M^{* *}=M_{R}^{* *}$. Since $M$ is an $R$-bimodule, so are $M^{*}$ and $M^{* *}$. Clearly, $M^{*}$ is a non-zero, finitely generated left $R$-module. However, $M^{*}$ might not be finitely generated as a right $R$-module. Nonetheless, suppose that $X$ is a finitely generated right $R$-submodule of $M^{*}$. Then Gabber's Theorem implies that

$$
r \text {-GK-dim }\left(\left(M^{*}+X_{R}^{* *}\right) / M^{*}\right) \leqslant \mathrm{GK}-\operatorname{dim}\left(X^{* *} / X\right) \leqslant \mu-2 .
$$

Thus, by [11, Proposition 5.6],

$$
\begin{aligned}
r-\mathrm{GK}-\operatorname{dim}\left(\left(X^{* *} M+R\right) / R\right) & \leqslant r-\mathrm{GK}-\operatorname{dim}\left(X^{* *} M / X M\right) \leqslant r-\mathrm{GK}-\operatorname{dim}\left(\left(X^{* *} / X\right) \otimes M\right) \\
& \leqslant r-\mathrm{GK}-\operatorname{dim}\left(X^{* *} / X\right) \leqslant \mu-2 .
\end{aligned}
$$

Thus, Corollary 2.4 implies that $X^{* *} M \subseteq R$ and $X^{* *} \subseteq M_{R}^{*}$.

(ii) In this case, $M^{* *}=M_{R}^{* *}$ is a finitely generated right $R$-module but may not be finitely generated as a left $R$-module. However, [11, Lemma 5.3(b)] and Gabber's Theorem imply that

$$
\ell-G K-\operatorname{dim}\left(M_{R}^{* *} / M\right) \leqslant r-\mathrm{GK}-\operatorname{dim}\left(\left(M_{R}^{* *}\right) / M\right) \leqslant \mu-2 .
$$

Thus, by Gabber's Theorem, again, $M_{R}^{* *} \subseteq{ }_{R} M^{* *}$. By symmetry, $M_{R}^{* *}={ }_{R} M^{* *}$.

One consequence of Corollary 2.5(ii) is that there is no ambiguity in saying that a finitely generated $R$-bisubmodule of $Q(R)$ is reflexive. Moreover, the maximal reflexive ideals of $R$ satisfy pleasant properties.

COROLlaRY 2.6. Let I be a proper, non-zero ideal of $R$. Then the following are equivalent:

(i) I is maximal among reflexive, proper ideals of $R$;

(ii) $I$ is maximal among ideals $J$ satisfying $\mathrm{GK}-\operatorname{dim}(R / J)=\mu-1$;

(iii) $I$ is a reflexive, height one prime ideal of $R$.

Proof. (i) $\Rightarrow$ (ii) If GK-dim $(R / I) \leqslant \mu-2$, then Gabber's Theorem implies that $I^{* *}=R$. On the other hand, if $J$ is an ideal such that $I \subseteq J$ and GK-dim $(R / J)=\mu-1$, then, by Gabber's Theorem and the exactness of Gelfand-Kirillov dimension, $\operatorname{GK}-\operatorname{dim}\left(R / J^{* *}\right)=\mu-1$. Hence $I=J^{* *}$, as required.

(ii) $\Rightarrow$ (iii) Once again, Lemma 2.1 and Theorem 2.2 imply that $I=I^{* *}$. Suppose that $A$ and $B$ are ideals of $R$, strictly containing $I$, and such that $A B \subseteq I$. Thus, GK-dim $(R / A) \leqslant \mu-2$ and $\mathrm{GK}-\operatorname{dim}(R / B) \leqslant \mu-2$. By the symmetry of Gelfand-Kirillov dimension,

$$
\mathrm{GK}-\operatorname{dim}(B / I) \leqslant \mathrm{GK}-\operatorname{dim}(B / A B) \leqslant \mathrm{GK}-\operatorname{dim}(R / A) \leqslant \mu-2 .
$$

Thus $B=I$ and $I$ is prime. 
(iii) $\Rightarrow$ (i) If $K$ is a proper ideal such that $K \supset I$ (where, again, $\supset$ is used in its strict sense), then [11, Proposition 5.1(e)] implies that

$$
\operatorname{GK}-\operatorname{dim}(R / K) \leqslant \mathrm{GK}-\operatorname{dim}(R / I)-1 \leqslant \mathrm{GK}-\operatorname{dim}(R)-2 .
$$

Thus, $K^{* *}=R$.

One should note that it is easy to construct examples of Auslander-regular, CM rings in which the height 1 prime ideals are not reflexive (for example, take $R=U\left(\mathfrak{I l}_{2}(\mathbb{C})\right) /(\Omega)$, where $\Omega$ is the Casimir element).

LEMma 2.7. Suppose that $R$ is not a maximal order. Then, possibly after replacing $R$ by its opposite ring $R^{\mathrm{op}}$, there exists a reflexive, height 1 prime ideal I of $R$ such that $S=\mathcal{O}_{\ell}(I) \supset R$.

Proof. By [14, Proposition 5.1.4], there exists a non-zero ideal $J$ of $R$ such that either $\mathcal{O}_{\ell}(J) \supset R$ or $\mathcal{O}_{r}(J) \supset R$. We shall assume that the former is the case, since the latter possibility is then covered by replacing $R$ by $R^{\text {op }}$. Now let $I$ be an ideal of $R$ maximal with respect to $\mathcal{O}_{\ell}(I) \supset R$ and set $S=\mathcal{O}_{\ell}(I)$. Note that, if $J={ }_{R} S^{*}$, then $S \cdot S J=S J \subseteq R$. Thus, $J=S J$ and the maximality of $I$ ensures that $I=J={ }_{R} S^{*}$. In particular, $I$ is reflexive (recall that Corollary 2.5(ii) implies that left and right reflexive are the same concept for ideals). Suppose next that $I$ is not prime, say $A B \subseteq I$, for some ideals $A, B$ that strictly contain $I$. Then $S A \nsubseteq R$, yet $(S A) B \subseteq I \subset B$. This yields the contradiction $\mathcal{O}_{\ell}(B) \supset R$. Thus, $I$ is prime and Corollary 2.6 implies that $I$ is of height one.

We need the following concept. Let $M$ be a finitely generated module over a Noetherian, Auslander-regular, CM ring $R$. Then GK-dim $(M)$ is an integer, by [13, Proposition 4.5]. If GK-dim $(M) \leqslant \alpha$, for some integer $\alpha$, then define $\varepsilon_{\alpha}(M)$ to be the maximal length of a chain of submodules of $M$ such that each factor has GK-dim equal to $\alpha$. Thus, by definition, $\varepsilon_{\alpha}(M)=0$ if and only if GK-dim $(M)<\alpha$. Moreover $\varepsilon_{\alpha}(M)$ is finite and $\varepsilon_{\alpha}$ is additive on short exact sequences (see [13, (4.6)]).

Naively, there are two ways of obtaining non-maximal orders. First, one can take an idealizer $B=\mathbb{\vartheta}_{A}(I)=\{a \in A: I a \subseteq I\}$ for a maximal left ideal $I$ of a ring $A$. In this case, $I$ is a reflexive right ideal of $B$ such that $I^{2}=I$. Note, however, that $A=I_{B}^{*}$ may not be finitely generated as a right $B$-module. Secondly, if $I$ is an ideal of a maximal order $A$ one could take $B=\{a \in A:[a+I] \in C / I\}$ for some proper subring $C / I$ of $A / I$. In this case, $A=I_{B}^{*}={ }_{B} I^{*}$ and so $A$ is a finitely generated $B$-bimodule. The next lemma shows that, in some sense, any Auslander-regular CM ring $R$ that is not a maximal order satisfies the properties of one of these two special cases. Modulo checking that certain homological conditions hold, the key idea in the proof of the theorem is then given by Lemma 2.9. Assume that $R$ is stably free, let $I$ be a reflexive prime ideal of $R$ and $M$ a finitely generated, reflexive right $R$-submodule of $Q(R)$. Then $M / M I$ is more or less isomorphic to $R / I$. Applying this to $M=I$ shows that $I^{2} \neq I$, implying that the first of our two possibilities cannot occur. Alternatively, if the ring $S=I_{R}^{*}$ of Lemma 2.7 is finitely generated as a right $R$-module, Lemma 2.9 implies that $S / R$ must be a torsion right $R / I$-module, contradicting Corollary 2.4 .

Leмma 2.8. Let $I$ be a reflexive, prime ideal of $R$ and assume that GK-dim $\left(I / I^{2}\right)=G K-\operatorname{dim}(R / I)$. Write $L={ }_{R} I^{*}$. Then $L I \subseteq R$. 
Proof. Set $\beta=\mu-1=\mathrm{GK}-\operatorname{dim}(R)-1$ and note that, by Corollary 2.6, GK-dim $(R / I)=\beta$. Let $A$ and $B$ be $R$-bimodules. Throughout the proof, $\operatorname{Hom}(A, B)$ will stand for the group of all $R$-homomorphisms from $A$ to $B$, regarded as left $R$-modules, while $\operatorname{Ext}^{p}(A, B)$ will stand for the corresponding homology group. First, consider the exact sequence

$$
\begin{aligned}
0 \longrightarrow \operatorname{Hom}(R / I, R / I) \longrightarrow & \operatorname{Hom}(R, R / I) \stackrel{\phi}{\longrightarrow} \operatorname{Hom}(I, R / I) \\
& \longrightarrow \operatorname{Ext}^{1}(R / I, R / I) \longrightarrow 0,
\end{aligned}
$$

and note that this is a sequence of $R$-bimodules. It is readily checked that $\phi=0$ and hence, again as $R$-bimodules,

$$
\operatorname{Ext}^{1}(R / I, R / I) \cong \operatorname{Hom}(I, R / I) \cong \operatorname{Hom}_{R / I}\left(I / I^{2}, R / I\right) .
$$

By hypothesis, $I / I^{2}$ is not torsion as a left module over the prime ring $R / I$. Thus, $\operatorname{Hom}_{R / I}\left(I / I^{2}, R / I\right)$ is non-zero and torsion-free as both a left and a right $R / I$-module (although it need not be finitely generated as a left module). Hence,

$$
\ell-\mathrm{GK}-\operatorname{dim}_{R}\left(\operatorname{Ext}^{1}(R / I, R / I)\right)=\mathrm{GK}-\operatorname{dim}_{R}(R / I)=\beta .
$$

Next, as $I$ is prime, $R / I$ is $\beta$-homogeneous. Thus, Lemma 2.3 implies that $r-\mathrm{GK}-\operatorname{dim}\left(\operatorname{Ext}^{p}(R / I, R)\right)<\beta$ for all $p>1$. Clearly, $\operatorname{Ext}^{0}(R / I, R)=0$.

These comments ensure that the hypotheses of Proposition 1.3(B) are satisfied for the case $M=N=R / I$ and $\alpha=\beta-1$ with $m=0$ and $n=1$. By that result,

$$
\ell-\mathrm{GK}-\operatorname{dim}_{R}\left(\operatorname{Ext}^{1}(R / I, R) \otimes R / I\right) \geqslant \beta .
$$

But, as $R$-bimodules, $\operatorname{Ext}^{1}(R / I, R) \otimes R / I \cong L / R \otimes R / I \cong L /(L I+R)$. Since $L$ is a finitely generated right $R$-module, this implies that

$$
r-\mathrm{GK}-\operatorname{dim}_{R}(L /(L I+R)) \geqslant \ell-\mathrm{GK}-\operatorname{dim}_{R}(L /(L I+R)) \geqslant \beta .
$$

Finally, suppose that $L I \nsubseteq R$. Then $I \subseteq(L I+R)_{R}^{*} \subset R$. Thus, by Corollary 2.6, $I=(L I+R)_{R}^{*}$ and $(L I+R)_{R}^{* *}=L$. But, by Theorem 2.2 , this implies that

$$
r-\mathrm{GK}-\operatorname{dim}(L /(L I+R)) \leqslant \mathrm{GK}-\operatorname{dim}(R)-2=\beta-1,
$$

giving the required contradiction.

As remarked in the Introduction, the main theorem can be proved under the hypothesis that the ring $R$ has trivial state space, rather than being stably free. This concept is defined as follows. Let $A$ be a Noetherian ring. Then, the state space $\operatorname{St}(A)$ is defined to be the set of all additive functions (states) $s$ from $K_{0}(A)$ to $\mathbb{R}$ such that $s([A])=1$ and $s([M]) \geqslant 0$ for all finitely generated projective right $A$-modules $M$. The ring $A$ has a trivial state space if $\mathrm{St}(\mathrm{A})$ consists of a unique function. A more convenient characterization of this is given as follows. Given a prime ideal $P$ of $A$ and a finitely generated right $A$-module $M$, set

$$
\hat{\rho}(M, P)=\frac{\text { length }_{Q(A / P)}(M / M P \otimes Q(A / P))}{\text { length }_{Q(A / P)}(Q(A / P))} .
$$

Then, [20, Theorem 6.4] implies that $A$ has a trivial state space if and only if $\hat{\rho}(M, P)=\hat{\rho}(M, Q)$, for all finitely generated, projective right $A$-modules $M$ and all prime ideals $P$ and $Q$. Since $\hat{\rho}(?, P)$ is additive on direct sums, it is clear that a stably 
free ring has trivial state space. The other obvious case when this condition holds is if $A$ is a prime ring in which every prime ideal is localizable (and so, in particular, it holds if $A$ is a commutative domain).

The significance of this concept to the results of this paper comes from the following lemma.

LEMMA 2.9. Assume that $R$ has a trivial state space and let $\beta$ be a positive integer. Let $K$ be a finitely generated, essential right $R$-submodule of $Q(R)$ and $N$ a finitely generated $R$-bimodule. Assume that $r-\mathrm{GK}-\operatorname{dim}_{R}\left(\operatorname{Tor}_{p}^{R}(K, N)\right) \leqslant \beta-1$ for all $p>0$ and that $\mathrm{GK}-\operatorname{dim}(N)=\beta$. Then $\varepsilon_{\beta}(K \otimes N)=\varepsilon_{\beta}(N)$.

Proof. Let $F$ be a finitely generated, projective right $R$-module. Then, GK-dim $(F \otimes N) \leqslant \beta$ and so $s(F)=\varepsilon_{\beta}(F \otimes N) / \varepsilon_{\beta}(N)$ is a non-negative real number. Since $\varepsilon_{\beta}(?, \otimes N)$ is additive on direct sums, $s$ defines a state. But, as $R$ is a prime ring with trivial state space, this implies that $s(F)$ equals the image of the other obvious state: $s(F)=\hat{\rho}(F, 0)$. In other words, $\varepsilon_{\beta}(F \otimes N)=\varepsilon_{\beta}(N) \hat{\rho}(F, 0)$.

Pick a finitely generated, projective resolution of $K_{R}$, say<smiles>O[Y]O[Te]CC[As][Te]O</smiles>

Since $R$ is a prime ring, $\hat{\rho}(?, 0)$ is additive on short exact sequences and so

$$
\sum_{j=0}^{d}(-1)^{j} \hat{\rho}\left(F^{j}, 0\right)=\hat{\rho}(K, 0)=1 .
$$

Consider the complex of right $R$-modules:

$$
0 \longrightarrow F^{d} \otimes N \longrightarrow \ldots \longrightarrow F^{0} \otimes N \longrightarrow K \otimes N \longrightarrow 0 .
$$

The homology groups of this complex are the modules $\operatorname{Tor}_{p}^{R}(K, N)$ for $p>0$ and, by hypothesis, $\varepsilon_{\beta}\left(\operatorname{Tor}_{p}^{R}(K, N)\right)=0$ for all $p>0$. Since $\varepsilon_{\beta}$ is additive on short exact sequences, evaluation of $\varepsilon_{\beta}$ on (2.9.2) therefore gives

$$
\varepsilon_{\beta}(K \otimes N)=\sum_{j=0}^{d}(-1)^{j} \varepsilon_{\beta}\left(F^{j} \otimes N\right) .
$$

By (2.9.1) and the comments of the first paragraph of the proof, this implies that

$$
\varepsilon_{\beta}(K \otimes N)=\sum_{j=0}^{d}(-1)^{j} \varepsilon_{\beta}\left(F^{j} \otimes N\right)=\varepsilon_{\beta}(N) \sum_{j=0}^{d}(-1)^{j} \hat{\rho}\left(F^{j}, 0\right)=\varepsilon_{\beta}(N),
$$

as required.

We briefly drop the standing assumption that $R$ is a prime ring.

THEOREM 2.10. Let $R$ be a Noetherian $k$-algebra and assume that $R$ is Auslanderregular and $\mathrm{CM}$. Then:

(i) if $R$ is stably free, then $R$ is a domain;

(ii) if $R$ is a prime ring with a trivial state space, then $R$ is a maximal order in its quotient division ring.

Proof. (i) The proof that $R$ is a domain is a mild generalization of [13, Theorem 4.8]. Let GK-dim $(R)=\mu$ and $\varepsilon\left(R_{R}\right)=t$. If $I$ is any non-zero right ideal of $R$, then $E^{0}(I) \neq 0$ and so the $\mathrm{CM}$ condition implies that $\mathrm{GK}-\operatorname{dim}(I)=\mu$. Equivalently, 
$0<\varepsilon_{\mu}(I) \leqslant t$. Moreover, by hypothesis, $I$ has a finite resolution by finitely generated, free right $R$-modules. Since $\varepsilon_{\mu}$ is additive on short exact sequences, this implies that $\varepsilon_{\mu}(I)$ is a multiple of $\varepsilon_{\mu}(R)=t$. Hence, $\varepsilon_{\mu}(I)=t$. But, if $R$ contains zero-divisors, then it is easy to see that $R$ contains a non-zero right ideal $J$ such that $\varepsilon_{\mu}(J)<t$ (see the proof of [13, Theorem 4.8]). Since no such right ideal $J$ can exist, $R$ must be a domain.

(ii) Suppose that $R$ is not a maximal order. Then, by Lemma 2.7, we may assume that there exists a reflexive, height 1 prime ideal $I$ of $R$ such that $S=\mathcal{O}_{\ell}(I) \supset R$. By Corollary 2.6, GK-dim $(R / I)=\mu-1$. We shall first prove that GK-dim $\left(I / I^{2}\right)=\mu-1$. Since ${ }_{R} M=I_{R}^{*}$ is reflexive, Lemma 2.3 implies that

$$
r-\mathrm{GK}-\operatorname{dim}\left(\operatorname{Ext}_{R}^{q}\left({ }_{R} M, R\right)\right) \leqslant \mu-3
$$

for all $q>0$. Trivially, $I={ }_{R} M^{*}$ is a finitely generated bimodule. Thus, the hypotheses of Proposition 1.3(C) are satisfied, in the case when $R=U=T, n=0$, $\alpha=\mu-3, N=R / I$ and $M=I_{R}^{*}$. Therefore, by that result,

$$
r-\mathrm{GK}-\operatorname{dim}\left(\operatorname{Tor}_{R}^{p}(I, R / I)\right) \leqslant \mu-3
$$

for all $p>0$. But this, in turn, implies that the hypotheses of Lemma 2.9 are satisfied for $K=I, \quad N=R / I$ and $\beta=\mu-1$. Thus, $\varepsilon_{\mu-1}\left(I / I^{2}\right)=\varepsilon_{\mu-1}(R / I)>0$ and hence $\operatorname{GK}-\operatorname{dim}\left(I / I^{2}\right)=\mu-1$.

Now, the left-handed version of Lemma 2.8 implies that $I S \subseteq I\left(I_{R}^{*}\right) \subseteq R$. Thus, $S$ is a finitely generated $R$-bimodule. We claim that this implies that $S=S_{R}^{* *}$. For, [11, Proposition 5.6] implies that

$$
\begin{aligned}
r-\mathrm{GK}-\operatorname{dim}\left(S_{R}^{* *} I / I\right) & =r-\mathrm{GK}-\operatorname{dim}\left(S_{R}^{* *} I / S I\right) \leqslant r-\mathrm{GK}-\operatorname{dim}\left(\left(S^{* *} / S\right) \otimes I\right) \\
& \leqslant r-\mathrm{GK}-\operatorname{dim}\left(S^{* *} / S\right) \leqslant \mu-2 .
\end{aligned}
$$

Thus, Gabber's Theorem implies that $S_{R}^{* *} I \subseteq I_{R}^{* *}=I$. Therefore, $S_{R}^{* *} \subseteq S$ and $S_{R}^{* *}=S$. Now repeat the argument of the last paragraph for the case $M=S_{R}^{*}$. Thus Proposition 1.3 implies that $r-\mathrm{GK}-\operatorname{dim}\left(\operatorname{Tor}_{R}^{p}(S, R / I)\right) \leqslant \mu-3$ for all $p>0$ and then Lemma 2.9 implies that $\varepsilon_{\mu-1}(S / I)=\varepsilon_{\mu-1}(S / S I)=\varepsilon_{\mu-1}(R / I)$. But, $\varepsilon_{\mu-1}$ is additive on short exact sequences. Thus, $\varepsilon_{\mu-1}(S / R)=0$; that is, $r-\mathrm{GK}-\operatorname{dim}_{R}(S / R)<\mu-1$. By Corollary 2.4 , this implies that $S=R$, as required.

COROllary 2.11. Let $R$ be as in Theorem 2.10 and suppose that $R$ satisfies $a$ polynomial identity. Then, in the notation of [14, Chapter 13.9], $R$ is equal to its own trace ring $T R$ and is integral over its centre $Z(R)$. Moreover, if $R$ is finitely generated as an algebra over some Noetherian central subring, then $R$ is a finite module over its centre $Z(R)$ and $Z(R)$ is a Noetherian maximal order.

\section{Proof. Use [14, Propositions 13.9.8 and 13.9.11].}

One unfortunate aspect of Theorem 2.10 is that it is unclear whether all the hypotheses of the theorem are required. However, easy examples given in Section 3 show that one does need to assume that $R$ is both Auslander-regular and has trivial state space. In contrast, it is a long-standing open question as to whether every Noetherian graded ring $R$ of finite global dimension is a domain and a maximal order. This question is obviously closely connected to the corresponding conjecture for local, as opposed to graded, rings. By [8] and [10], this second conjecture is true for local rings that are integral over their centres, and this easily implies the result for graded rings. 
Proposition 2.12. Let $R$ be a graded Noetherian ring of finite global dimension, such that $R$ is integral over its centre. Then $R$ is a domain and is a maximal order in its quotient division ring.

Proof. Write $R=\bigoplus_{j \geqslant 0} R_{j}$; thus, by definition, $R=\mathbf{m}+k$, where $\mathbf{m}=\bigoplus_{j \geqslant 1} R_{j}$ is the graded radical of $R$ and $k=R_{0}$ is a central subfield of $R$. If $r \in R$, we shall always write $r=r_{a}+r_{a+1}+\ldots+r_{b}$, where the $r_{j} \in R_{j}$ are the homogeneous components. Given that $r_{b} \neq 0$, then $r_{b}=\sigma(r)$ is called the principal symbol of $r$.

If $Z(R)$ denotes the centre of $R$, set $\mathbf{n}=\mathbf{m} \cap Z(R)$ and $\mathscr{C}=Z(R) \backslash \mathbf{n}$. Since $R$ is integral over $Z(R)$, 'going up' and 'incomparability' imply that $\mathbf{n}$ is a maximal (and graded) ideal of $Z(R)$ and that $R / \mathrm{n} R$ is a graded, artinian ring (see [14, Theorem 13.8.14]). Thus, $\mathbf{m}^{r} \subseteq \mathbf{n} R$, for some $r$. Consequently, 'going up' implies that $R_{\mathscr{Q}}$ is a local ring with Jacobson radical $\mathbf{m}_{\mathscr{C}}$. Note that $R_{\mathscr{C}} / \mathbf{m}_{\mathscr{C}} \cong k$ is a field. Of course, $R_{\mathscr{C}}$ is still integral over its centre $Z(R)_{\mathscr{B}}$ and $R_{\mathscr{C}}$ still has finite global dimension. Hence, by [8, Theorem 6.7(i)] and [7, Corollary 3.4], $R_{\mathscr{C}}$ is a domain and, by [10], $R_{\mathscr{C}}$ is a maximal order.

If $R$ is not a domain then $a c=0$ for some non-zero elements $a \in R$ and $c \in \mathscr{C}$. But, $c=c_{0}+c_{1}+\ldots+c_{v}$, where $c_{0} \neq 0$. If $a=a_{r}+\ldots+a_{s}$ then $a c=0$ forces $c_{0} a_{r}=0$. Since $c_{0} \in k$ is a unit, this implies that $a=0$, a contradiction. Thus $R$ is a domain.

Now assume that $R$ is not a maximal order. Then there exists a ring $S$ with $Q(R) \supseteq S \supset R$ such that $S I=I$ (or $I S=I$ ) for some non-zero ideal $I$ of $R$. Fix a nonzero element $d \in Z(R) \cap I$ and set $V=d S \supset d R$. Since $V I=d I$, it follows that

$$
\sigma(V) \sigma(I) \subseteq \sigma(V I)=\sigma(d I)=\sigma(d) \sigma(I) .
$$

Similarly, $\sigma(V) \supset \sigma(d) R$. Thus, $\mathcal{O}_{\ell}(\sigma(I)) \supseteq \sigma(d)^{-1} \sigma(V) \supset R$. Pick $h=h_{n} \in \sigma(V) \cap R_{n}$, for some $n$, such that $\sigma(d)^{-1} h \notin R$. Since $R_{\mathscr{C}}$ is a maximal order, $\mathcal{O}_{\ell}(\sigma(I)) \subseteq R_{\mathscr{C}}$ and $h c \in \sigma(d) R$, for some $c=c_{0}+\ldots+c_{t} \in \mathscr{C}$. But, $c_{0} \neq 0$ is a central unit in $R$. Thus, if $h c=\sigma(d) t$, and $\sigma(d) \in R_{u}$, then $h=\sigma(d) c_{0}^{-1} t_{n-u} \in \sigma(d) R$, contradicting the choice of $h$. Thus, $R$ is indeed a maximal order.

\section{Examples}

We end with a few examples to illustrate some aspects of the results of this paper. The examples we use are not new, indeed they are all familiar for one reason or another. First, one might hope that the assumption that $R$ be an Auslander-regular, CM ring is enough by itself to ensure that $R$ is a maximal order. However, this is easily seen not to be the case.

LEMMA 3.1. (i) Let $R$ be an hereditary, Noetherian prime (HNP) ring. Then $R$ is Auslander-regular.

(ii) Let $R$ be an HNP ring that satisfies a polynomial identity and is finitely generated as an algebra over a central subfield $k$. Then $R$ is also CM.

Proof. (i) Localization commutes with the taking of homology. Thus, for any finitely generated (left) $R$-module $M$,

$$
\operatorname{Ext}_{R}^{1}(M, R) \otimes_{R} Q(R) \cong \operatorname{Ext}_{Q(R)}^{1}(M \otimes Q(R), Q(R))=0 .
$$

Thus, $\operatorname{Ext}_{R}^{1}(M, R)$ is a torsion right $R$-module and $\operatorname{Ext}^{0}(N, R)=0$ for any submodule $N$ of $\operatorname{Ext}_{R}^{1}(M, R)$. This is all that is required to prove that $R$ is Auslander-regular. 
(ii) By the Nullstellensatz [14, Proposition 13.10.6], GK-dim $(R)=1$ and a finitely generated $R$-module $M$ is torsion if and only if it is finite dimensional over $k$. Thus, $j(M)=1 \Leftrightarrow M$ is torsion $\Leftrightarrow \mathrm{GK}-\operatorname{dim}(M)=0$.

By Lemma 3.1, an explicit example of an Auslander-regular, CM ring that is not a maximal order is

$$
\left(\begin{array}{cc}
k[x] & x k[x] \\
k[x] & k[x]
\end{array}\right) .
$$

Similarly, it is easy to construct Auslander-regular rings that are not CM. Indeed, if $A=\mathbb{C}[x, \partial / \partial x]$ is the first Weyl algebra, then the ring $B=\mathbb{C}+x A$ is an HNP ring (and hence Auslander-regular), by [14, Example 5.5.5]. However, $B$ is not CM since it has two simple right modules $A / B$ and $B / x A$ of Gelfand-Kirillov dimensions 1 , respectively 0 . Nonetheless, it is possible to modify this construction to obtain a Cohen-Macaulay example.

EXAMPLE 3.2. There exists an hereditary, Auslander-regular, $\mathrm{CM}$ domain $R$ and $a$ maximal ideal $I$ of $R$ such that $S=\mathcal{O}_{\ell}(I) \supset R$ yet $S_{R}$ is not finitely generated.

Proof. Let $D_{i}$ be a division ring, $p_{i}^{2}$-dimensional over its centre $\mathbb{Q}$, where the $p_{i}$ run through the integer primes. Let $D=D_{1} \otimes_{Q} D_{2} \otimes_{Q} D_{3} \ldots$ be the Krull division ring. Identify each $D_{i}$ with its image in $D$ and let $F_{i}=\mathbb{Q}\left(d_{i}\right)$ be a maximal subfield of $D_{i}$. Then there is an automorphism $\sigma$ of $D$ that acts on $D_{i}$ by conjugation by $d_{i}$. Clearly, no power of $\sigma$ is inner and so the skew group ring $S=D\left[x, x^{-1} ; \sigma\right]$ is a simple ring (see, for example, [14, Theorem 1.8.5]). Clearly, $S(x-1)$ is a maximal left ideal of $S$, so set

$$
R=\mathbb{\square}_{S}(S(x-1))=F+S(x-1) \supset I=S(x-1),
$$

where $F=\otimes F_{i}$. Thus, by [14, Theorem 5.5.8], $R$ is an HNP ring. Since $D$ is locally finite dimensional over $k=\mathbb{Q}$, it follows that

$$
\mathrm{GK}-\operatorname{dim}(D)=0 \quad \text { and } \quad \mathrm{GK}-\operatorname{dim}(R)=\mathrm{GK}-\operatorname{dim}(S)=1 .
$$

Consequently, every simple $R$-module has Gelfand-Kirillov dimension zero. Thus, $R$ is Auslander-regular and CM, by (the proof of) Lemma 3.1. However, as a right module over $R$ or $R / I$, we have that $S / I \cong D_{F}$ is an infinite direct sum of simple modules. Since $S / I$ has length two as a left $R$-module, it follows easily that there is no proper over-ring of $R$ that is a finitely generated $R$-bimodule.

We remark that there does not exist a finitely generated $k$-algebra with the properties of Example 3.2. Indeed, the techniques of [12, Theorem 2] can be used to prove the following fact. Let $R$ be a Noetherian, Auslander-regular, CM domain that is an affine $k$-algebra. Assume that $R$ is not a maximal order and let $S$ be defined by Lemma 2.7. Then $S$ is a finitely generated $R$-bimodule. Moreover, $S_{R}^{* *}=S$.

The next example shows that the main theorem will also fail if one does not assume that the ring $R$ is Auslander-regular.

EXAMPLE 3.3. There exists a Noetherian domain $R$, with $\operatorname{gldim}(R)=3$, such that $R$ is not a maximal order.

Proof. Let $S=U(\mathrm{~g})$ be the enveloping algebra of the two-dimensional complex 
solvable Lie algebra $g$; thus $S$ has generators $x$ and $y$ subject to the relation $x y-y x=x$. Set $R=\mathbb{C}+y S$. Then, by [19, Section 3], $R$ is an affine Noetherian ring, with $\operatorname{gldim}(R)=3$ and $K_{0}(R)=\mathbb{Z}$. By Theorem $2.10, R$ cannot be both Auslanderregular and CM. In fact, it is not Auslander-regular. To see this, note that $P_{1}=y S \supset P_{2}=y x S$ are non-zero prime ideals of $R$, and $P_{1}$ is reflexive as a left ideal. Thus, Corollary 2.6 implies that $R$ is not Auslander-regular.

Finally, it is reasonable to suppose that, in the PI case, the following stronger version of Theorem 2.10 should hold.

QUESTION 3.4. Let $R$ be a stably free, Noetherian, Auslander-regular ring that satisfies a PI. Then, is $R$ a maximal order?

Given such a ring $R$, then the argument of Theorem 2.10 shows that $R$ is a domain and standard localization techniques allow one to reduce to the case where $R$ is a semilocal ring of Krull dimension one. Such a ring will also be a finite module over its centre. The theorem would then be very easy to prove if one could prove the following: if $R$ is an Auslander-regular ring such that $R$ is a finite module over its centre $Z(R)$, and $\mathrm{K}-\operatorname{dim}(R)=1$, then $R$ is an HNP ring. For, in such a case, known results about the K-theory of HNP rings would imply that, if $R$ is stably free, then $R$ is a Dedekind domain. However, as the next example shows, this last question is false. To save repetition, a ring $A$ will be called a classical order if $A$ is a prime ring that is finitely generated as a module over its centre $Z(A)$ and such that $Z(A)$ is a Noetherian domain of Krull dimension one.

EXAMPLE 3.5. There exists a semilocal, classical order $R$ such that $R$ is an Auslander-regular ring but $\operatorname{gldim}(R)=2$ and $\operatorname{gldim}(Z(R))=\infty$.

Proof. The example is a standard example of a ring of finite global dimension that has a centre of infinite global dimension. Set $C=k[x]_{(x)}$ and let

Set

$$
R=\left(\begin{array}{cc}
k+x^{2} C & x^{2} C \\
C & C
\end{array}\right) \subset M_{2}(C) .
$$

$$
P=\left(\begin{array}{cc}
x^{2} C & x^{2} C \\
C & C
\end{array}\right) \text { and } Q=\left(\begin{array}{cc}
k+x^{2} C & x^{2} C \\
C & x C
\end{array}\right)
$$

It is readily checked that $R$ is a finite module over its centre $Z(R)=k+x^{2} C$, and that $P$ and $Q$ are maximal ideals of $R$. Moreover, $(P \cap Q)^{3} \subset x^{2} R$ and so $P$ and $Q$ are the only maximal ideals of $R$. Since $P$ is a generative right ideal of $M_{2}(C),[14$, Theorem 7.5.13] implies that

$$
\operatorname{gldim}(R) \leqslant \operatorname{gldim}\left(M_{2}(C)\right)+\operatorname{gldim}(R / P)+1=2 .
$$

Another easy exercise shows that $Q$ is not reflexive as either a left or a right ideal of $R$. Hence, $Q$ is not projective and $\operatorname{gldim}(R)=2$. Note, however, that $P$ is reflexive as both a left and a right ideal and so $P$ is projective. Finally, we need to prove that $R$ is Auslander-regular. As gldim $(R)=2$, an easy induction on the length of a torsion $R$-module shows that one need only prove that $\operatorname{Ext}_{R}^{1}(N, R)=0$ when $N$ is a 
submodule of $\operatorname{Ext}_{R}^{2}(M, R)$ for a simple (right) $R$-module $M$. Note that $R$ has just two simple modules, and one of them, $R / P$, has $\operatorname{hd}(R / P)=1$. Thus, one need only consider the case when $M=R / Q$. Let

$$
\alpha=\left(\begin{array}{ll}
1 & 0 \\
0 & x
\end{array}\right) \text { and } \beta=\left(\begin{array}{ll}
1 & 0 \\
1 & x
\end{array}\right) .
$$

Then straightforward computations prove that $Q=\alpha R+\beta R$ while $\beta^{-1} \alpha R \cap R=P$. This provides a short exact sequence (of right modules)

$$
0 \longrightarrow P \stackrel{\sigma}{\longrightarrow} R \oplus R \stackrel{\tau}{\longrightarrow} Q \longrightarrow 0,
$$

where $\sigma(p)=\left(\alpha^{-1} \beta p,-p\right)$ and $\tau\left(r_{1}, r_{2}\right)=\alpha r_{1}+\beta r_{2}$. Now, apply Hom(?, $\left.R\right)$ to obtain the exact sequence

$$
Q_{R}^{*} \longrightarrow\left(R^{(2)}\right)^{*} \stackrel{\phi}{\longrightarrow} P_{R}^{*} \longrightarrow E^{1}(Q) \longrightarrow 0 .
$$

Here, $\phi\left(r_{1}, r_{2}\right)=r_{1}+r_{2}\left(\alpha^{-1} \beta\right)$. It follows that

$$
P_{R}^{*}=\left(\begin{array}{cc}
C & x^{2} C \\
x^{-2} C & C
\end{array}\right) \text { and } \operatorname{Im}(\phi)=\left(\begin{array}{cc}
C & x^{2} C \\
x^{-1} C & C
\end{array}\right) .
$$

This implies that $E^{2}(R / Q) \cong E^{1}(Q) \cong P^{*} / \operatorname{Im}(\phi)$ is a simple left $R$-module. Since $Q P^{*} \subset \operatorname{Im}(\phi)$ we have $E^{2}(R / Q) \cong(R / Q)$. Since $Q$ is not a reflexive left ideal, $j\left({ }_{R} R / Q\right)=2$ and so $E^{12}(Q) \cong E^{1}\left({ }_{R} R / Q\right)=0$. Since $E^{2}\left({ }_{R} R / Q\right)$ is simple this completes the proof.

It is possible to find domains that satisfy the properties of Example 3.5, but the computations for such examples are less transparent. For example, let $S=\mathbb{H}[x]$, where $\mathbb{H}=\mathbb{R}[i, j, k]$ denotes the quaternions, and set $R^{\prime}=\mathbb{C}+(\mathrm{i} x+1)^{2} S$. By mimicking the above proof, one can show that $R^{\prime}$ satisfies the conclusion of Example 3.5. Since

$$
\begin{aligned}
& R^{\prime} /\left(x^{2}+1\right)^{2} R^{\prime} \cong\left(R^{\prime} /(\mathrm{i} x+1)^{2} R^{\prime}\right) \oplus\left(R^{\prime} /(\mathrm{i} x+1)^{2} R^{\prime}\right), \\
& R^{\prime} /\left(x^{2}+1\right) R^{\prime} \cong M_{2}(\mathbb{C}),
\end{aligned}
$$

it is probably not surprising that the properties of $R$ and $R^{\prime}$ are similar.

By repeating this process for the ring

$$
R^{\prime \prime}=\left(\begin{array}{ccc}
C & x C & x^{2} C \\
C & C & x C \\
C & C & C
\end{array}\right)
$$

one finds that $R^{\prime \prime}$ is a semilocal, Auslander-regular ring, again of global dimension two but now such that $Z\left(R^{\prime \prime}\right)=C$ is hereditary. Note, however, that none of these examples is $\mathrm{CM}$, since in each case the ring has (at least) two simple modules, necessarily of Gelfand-Kirillov dimension zero, but of differing homological dimension.

In fact, rings satisfying the hypotheses of Example 3.5 cannot be $\mathrm{CM}$ and this can be used to give a considerably easier proof of Theorem 2.10, in the case of PI rings. The details of this are left to the reader, but the idea is as follows. We are assuming that $R$ is a stably free, Auslander-regular, CM, Noetherian, prime PI ring. Assume 
that $R$ is not a maximal order and, as in Section 2, pick a reflexive prime ideal $I$ of $R$ such that $S=\mathcal{O}_{l}(I) \supset R$. As $I$ is of height one, one may localize at the clique of $I$. Standard ring theory ensures that the localized ring still satisfies the hypotheses of $R$, except that it now has Krull dimension one. Thus, we may assume that $R$ has Krull dimension one, and hence is a classical order. As $R$ is stably free but not a maximal order, it cannot be hereditary. The crucial point is that all prime ideals $P_{i}$ of $R$ that are in the same clique must satisfy

$$
\operatorname{GK}-\operatorname{dim}\left(R / P_{i}\right)=\mathrm{GK}-\operatorname{dim}\left(R / P_{j}\right)
$$

for all $i$ and $j$. Thus, we may pick a maximal ideal $P$ such that $P$ is not reflexive (and hence $j(R / P) \geqslant 2$ ). Now localize at the clique of $P$. This gives one a new Auslanderregular, CM classical order $R^{\prime}$ that is not hereditary and had no reflexive prime ideals. By Lemma $2.7, R^{\prime}$ is therefore a maximal order and this contradicts [14, Theorems 5.3.13 and 5.3.16].

It is easy to show that the ring defined by Example 3.5 is not stably free (and this is also true for the domain $R^{\prime}$ constructed after that example). In fact, apart from the Dedekind domains, we know of no semilocal, classical order $S$ for which $K_{0}(S)=\mathbb{Z}$. If no such ring can exist, then Question 3.4 can be easily answered in the positive.

Note added in proof. Since this paper was accepted, it has been proved that, if $R$ is any fully bounded Noetherian (FBN) graded ring of finite global dimension, then $R$ is Auslander-regular and CM (see [21]). The corresponding result for local FBN rings has been proved in [22]. These papers therefore answer, for FBN rings and hence for PI rings, the questions raised at the end of the Introduction and prior to Proposition 2.12.

\section{References}

1. M. ARTIN, 'Geometry of quantum planes', Azumaya algebras, actions and modules, Contemporary Mathematics 124 (Eds. D. Haile and J. Osterberg; American Mathematical Society, Providence, 1992).

2. M. Artin and W. Schelter, 'Graded algebras of global dimension 3', Adv. Math. 66 (1987) 171-216.

3. M. ARtin, J. TAte and M. VAN DEN BERGH, 'Some algebras associated to automorphisms of curves', The Grothendieck Festschrift I, Progress in Mathematics 86 (Eds. P. Cartier et al.; Birkhauser, Boston, 1990) 33-85.

4. M. Artin, J. TAte and M. van Den BerGh, 'Modules over regular algebras of dimension 3', Invent. Math. 106 (1991) 335-388.

5. J. E. BJöRK, Rings of differential operators (North-Holland, Amsterdam, 1979).

6. J. E. BJöRK and E. K. EKSTRÖM, 'Filtered Auslander-Gorenstein rings', Colloque en l'honneur de J. Dixmier (Birkhauser, 1990).

7. K. A. Brown, C. R. Hajarnavis and A. B. MacEacharn, 'Noetherian rings of finite global dimension', Proc. London Math. Soc. 44 (1982) 349-371.

8. K. A. Brown, C. R. Hajarnavis and A. B. MacEacharn, 'Rings of finite global dimension integral over their centres', Comm. Algebra 11 (1983) 67-93.

9. H. Cartan and S. Eilenberg, Homological algebra (Princeton University Press, 1956).

10. A. J. GRAY, 'A class of maximal orders integral over their centres', Glasgow Math. J. 24 (1983) 177-180.

11. G. KRAUSE and T. H. LenAGAN, Growth of algebras and Gelfand-Kirillov dimension (Pitman, London, 1985).

12. T. H. LENAGAN, 'Failure of the Nullstellensatz influences the growth of affine algebras', Bull. London Math. Soc. 17 (1985) 549-553.

13. T. LeVASSEUR, 'Some properties of non-commutative regular rings', Glasgow Math. J. 34 (1992) 277-300.

14. J. C. MCCONNELl and J.C. Robson, Non-commutative Noetherian rings (Wiley-Interscience, Chichester, 1987). 
15. J. J. Rotman, An introduction to homological algebra (Academic Press, New York, 1979).

16. S. P. SMith, 'The four dimensional Sklyanin algebra at points of finite order', preprint (1992).

17. S. P. SMITH and J. T. STAFFORD, 'Regularity of the four-dimensional Sklyanin algebra', Compositio Math. 83 (1992) 259-289.

18. J. T. STAFFORD, 'Regularity of algebras related to the Sklyanin algebra', Trans. Amer. Math. Soc. 341 (1994) 895-916.

19. J. T. STAFFORD, 'Global dimension of semiprime Noetherian rings', Séminaire Malliavin, Lecture Notes in Mathematics 1296 (Springer, Berlin-New York, 1987).

20. J. T. STAFFORD, 'Generating modules efficiently: Algebraic K-theory for non-commutative Noetherian rings', J. Algebra 69 (1981) 312-346.

21. J. T. STAFFORD and J. J. ZHANG, 'Homological properties of (graded) Noetherian PI rings', J. Algebra, to appear.

22. K-M. TEO, 'Homological properties of fully bounded Noetherian rings', preprint, University of Michigan, 1994.

Department of Mathematics

University of Michigan

Ann Arbor

Michigan 48109

USA

E-mail: jts@math.lsa.umich.edu 